\title{
Modelagem de carbono orgânico em rios urbanos: aplicabilidade para o planejamento e gestão de qualidade de água
}

\section{Organic carbon simulation in polluted rivers: applicability in water quality planning and management}

\author{
Heloise Garcia Knapik ${ }^{1}$, Cristovão Vicente Scapulatempo Fernandes ${ }^{1}$ e Júlio César Rodrigues de Azevedo ${ }^{2}$ \\ ${ }^{1}$ Universidade Federal do Paraná, Curitiba, PR, Brasil \\ ${ }^{2}$ Universidade Tecnológica Federal do Paraná, Curitiba, PR, Brasil \\ E-mails: heloise.dhs@ufpr.br (HGK),cris.dhs@ufpr.br (CVSF),jcrazevedo@utfpr.edu.br (JCRA)
}

Recebido: Janeiro 19, 2016 - Revisado: Junho 30, 2016 - Aceito: Agosto 11, 2016

\begin{abstract}
RESUMO
O objetivo do presente estudo é o de analisar a aplicabilidade de um modelo de transporte e decaimento de carbono orgânico para a simulação da qualidade da água em rios urbanos como subsídio para atividades de planejamento e gestão de recursos hídricos. A abordagem conceitual baseia-se na hipótese de que as frações particulada, dissolvida, lábil e refratária de carbono orgânico, que diferem na sua composição em função de sua origem no sistema, apresentando distintos mecanismos de degradação e, consequentemente, no impacto em termos da depleção da concentração de oxigênio dissolvido na coluna d'água. O desenvolvimento do trabalho consistiu na determinação quantitativa e qualitativa das frações de carbono orgânico no rio Iguaçu, localizado na Bacia do Alto Iguaçu, e no desenvolvimento dos algoritmos de simulação e calibração automática do modelo proposto, denominado ROCS - Model (River Organic Carbon Simulation Model). O modelo foi estruturado em planilhas Excel, com programação estruturada em VBA (Visual Basic for Applications), considerando escoamento unidimensional em condições de regime permanente em rios. O modelo considera múltiplas entradas de cargas difusas e pontuais. A calibração é feita através de um algoritmo de otimização por colônias de partículas (PSO), utilizando como estudo de caso dados de monitoramento de qualidade da água em 6 estações de controle ao longo de $107 \mathrm{~km}$ do rio principal, no Rio Iguaçu, localizado em uma área de intensa urbanização da região de Curitiba- PR, com aproximadamente 3 milhões de habitantes em uma área de $3.000 \mathrm{~km}^{2}$. Os resultados indicam que a presente abordagem, considerando a simulação das frações de carbono orgânico, tem potencial para ser aplicado em modelos de gestão de recursos hídricos, como alternativas aos parâmetros de qualidade da água tradicionais previstos na legislação Brasileira.
\end{abstract}

Palavras-chave: Carbono orgânico; Modelagem matemática; Gestão de qualidade de água; Rios urbanos.

\begin{abstract}
The main goal of this paper was to develop and evaluate the applicability of an organic carbon simulation model to simulate the water quality in polluted rivers. The methodological approach is based on the hypothesis that different fractions of organic carbon, such as particulate, dissolved, labile and refractory, can differently impact in the oxygen depletion due its origin and composition, and. The study was conducted at Iguassu River, located in Upper Iguassu Watershed, with a quantitative and qualitative monitoring of organic carbon fractions, the model and the automatic calibration routine development. The model was implemented in Excel spreadsheet and VBA language, considering a one-dimensional and permanent flow. Multiple diffuse and point sources can be considered during the simulation. The calibration is based on Particle Swarm Optimization algorithm, with 6 control stations in $107 \mathrm{~km}$ river length. The case study was developed at Iguassu River, located in a high urbanized basin in Curitiba-PR, an area about $3.000 \mathrm{~km}^{2}$ with approximately 3 million people. The results indicated that the current approach considering distinct organic carbon fractions simulations have potential to be applied in water resources planning and management models.
\end{abstract}

Keywords: Organic carbon; Mathematical modelling, Water quality management; Urban rivers. 


\section{INTRODUÇÃO}

Para uma efetiva gestão dos recursos hídricos, em especial em rios em bacias urbanas e com intensa degradação ambiental, o uso conjunto de um plano de monitoramento quali-quantitativo e de modelos matemáticos de simulação são estratégias essenciais para o estudo e avaliação da evolução temporal e espacial da qualidade da água. Dentro deste contexto, a representatividade dos parâmetros de interesse, tanto para fins de monitoramento, modelagem e fiscalização, deve ser analisada com cautela. A concentração de oxigênio dissolvido (OD) e a demanda bioquímica de oxigênio (DBO) são os parâmetros tradicionalmente utilizados pelo gestor, cujos diferentes níveis condicionam a existência ou não de seres vivos no ambiente aquático (THOMAS; THERAULAZ, 2007; JOUANNEAU et al., 2014). A DBO está relacionada ao conteúdo de matéria orgânica biodegradável, enquanto a concentração de OD é o elemento fundamental nos processos de oxidação e estabilização da matéria orgânica, sendo produzido tanto via fotossíntese, como por processos de trocas na interface ar-água.

$\mathrm{Na}$ questão da gestão de recursos hídricos, os atuais modelos matemáticos utilizados são baseados, principalmente, na simulação da relação OD-DBO (SHANAHAN et al., 1998), como, por exemplo, o mundialmente utilizado Qual2E (BROWN; BARNWELL JUNIOR, 1987). Contudo, a determinação da DBO é baseada em um ensaio analítico subjetivo, com limitações e de difícil interpretação. Ainda, sua determinação limita-se à fração biodegradável, não sendo possível inferir sobre a composição do material orgânico presente (COMBER; GARDNER; GUNN, 1996; JOUANNEAU et al., 2014). No ecossistema aquático, a matéria orgânica é parte essencial da cadeia alimentar e no processo de ciclagem de nutrientes. Faz parte de sua constituição uma complexa mistura de compostos orgânicos de diferentes origens, nas formas dissolvida, particulada, nos sedimentos e na biota aquática, o que lhe confere a característica de apresentar diferentes níveis de refratabilidade e, consequentemente, distintos mecanismos de transporte e degradação no ambiente aquático (MOSTOFA et al., 2013).

O carbono orgânico, por sua vez, analisado nas suas formas dissolvida (DOC), particulada (POC) e total (TOC), vem sendo atualmente indicado como o parâmetro mais relevante para a determinação global da matéria orgânica em ambientes aquáticos (LEENHEER; CROUÉ, 2003; THOMAS; THERAULAZ, 2007). Sua análise foi proposta ainda na década de 70 como uma alternativa às incertezas e dificuldades nos ensaios para a determinação da DBO.

Adicionalmente, a segmentação em função da fração dissolvida e da fração particulada, que diferem na sua composição em função de sua origem, permite avaliar os diferentes mecanismos de degradação e o respectivo consumo de oxigênio (KNAPIK et al., 2015). A associação com análises de espectroscopia de fluorescência e de ultravioleta visível também permitem uma análise qualitativa da matéria orgânica, com a classificação entre o que é refratário e o que é lábil no sistema (KNAPIK et al., 2014).

Contudo, o carbono orgânico ainda não é contemplado como variável para a representação da matéria orgânica pela maioria dos modelos de qualidade de água (SHANAHAN et al., 1998; CHAPRA, 1999), em especial aqueles aplicados em planejamento e gestão de bacias urbanas (KNAPIK, 2014). De acordo com os autores, a composição do carbono orgânico em função de sua origem no sistema pode influenciar distintos aspectos de planejamento e gestão de recursos hídricos. A não inclusão desse parâmetro nos modelos historicamente desenvolvidos era em função da dificuldade de sua determinação analítica. Atualmente, contudo, tanto a determinação direta da concentração das frações de carbono orgânico, como do uso de técnicas complementares, como a espectroscopia de fluorescência e de ultravioleta visível, já permite a análise rápida, com menor subjetividade e incerteza (KNAPIK et al., 2014).

Diferentes estudos apresentam metodologias e resultados para a caracterização e modelagem do fluxo de carbono orgânico em ecossistemas aquáticos (KARLSSON; RICHARDSON; KIFFNEY, 2005), com foco no aporte de carbono orgânico via escoamento superficial (OUYANG, 2003; EVANS; MONTEITH; COOPER, 2005), modelagem distribuída integrando processos do ciclo do carbono no solo e o balanço na água (SORRIBAS et al., 2012) e na dinâmica temporal em sistemas lacustres e/ou reservatórios (WESTPHAL; CHAPRA; SUNG, 2004; WEISSENBERGER et al., 2010) e oceanos (MOPPER et al., 1991), em ambientes com pouca ou nenhuma influência antrópica.

Modelos como o Qual2K (CHAPRA; PELLETIER, 2003), CE-Qual-W2 (COLE; WELLS, 2008), Aquatox (CLOUGH, 2014) e Delft 3D (DELTARES, 2014), consideram diferentes frações de constituintes relacionados à matéria orgânica de forma complementar à simulação do balanço OD-DBO, avançando em aspectos não considerados em modelos mundialmente difundidos, como o Qual2E (BROWN; BARNWELL JUNIOR, 1987). Por exemplo, o modelo Qual2K considera a separação entre DBO de degradação lenta e rápida (assumindo que a determinação foi realizada em amostras filtradas) e matéria orgânica particulada (representada por detritos e fitoplancton) (CHAPRA; PELLETIER, 2003; KNAPIK et al., 2011). O modelo CE-Qual-W2 (COLE; WELLS, 2008) permite a simulação de carbono orgânico como sendo uma fração da matéria orgânica na água em sedimentos. Já os modelos Aquatox (CLOUGH, 2014) e Delft 3D (DELTARES, 2014) recentemente incorporaram em seus processos formulações para simular a dinâmica da matéria orgânica em ambientes aquáticos. Contudo, os modelos apresentados são complexos, com uma demanda significativa de dados de entrada e coeficientes necessários à adequada calibração, os quais em geral não são considerados em estratégias de monitoramento pelos órgãos gestores de recursos hídricos (KNAPIK, 2014). Consubstancia, neste aspecto, uma lacuna na literatura de um modelo de qualidade da água que permita avaliar condições da dinâmica da matéria orgânica em rios urbanos, que seja de fácil implementação e avaliação, e baseado em parâmetros menos subjetivos que a DBO, conforme destacado em Knapik (2014).

Dentro deste contexto, o presente estudo teve como objetivo desenvolver, implementar e testar um modelo simplificado para o balanço e transporte de carbono orgânico aplicado a um estudo de caso em um rio urbano. O monitoramento e a aplicação do modelo foi realizado para um trecho do Rio Iguaçu na Bacia do Alto Iguaçu, englobando a cidade de Curitiba e Região Metropolitana. No que concerne ao monitoramento quali-quantitativo, foram consideradas 6 estações ao longo de $107 \mathrm{~km}$ do rio, contemplando áreas com baixa ocupação antrópica (cabeceira), intensa atividade poluidora 
(região central) e áreas em estágio de recuperação. Neste artigo, destacam-se a abordagem metodológica e os principais resultados da calibração e das simulações da concentração de carbono orgânico com o modelo desenvolvido, tendo como estudo de caso uma bacia urbana. Adicionalmente, uma abordagem original destacando a necessidade de uma nova visão quali-quantitativa para a gestão de recursos hídricos.

\section{MATERIAIS E MÉTODOS}

\section{Área de estudo e planejamento experimental}

Para as atividades de monitoramento de campo e avaliação da resposta dos resultados de simulação do modelo desenvolvido, foram considerados $107 \mathrm{~km}$ do rio Iguaçu, na Região Metroplitana de Curitiba. Dentro do contexto do Estado do Paraná, a Bacia do Alto Iguaçu figura como uma importante bacia tanto para fins de abastecimento, como para disposição final de efluentes domésticos e industriais. Com uma área aproximada de $3.000 \mathrm{~km}^{2}$, drena a cidade de Curitiba e Região Metropolitana, englobando um montante de aproximadamente 3 milhões de habitantes. Por ser uma região de cabeceira com intenso desenvolvimento urbano, possui um grande número de tributários em condições precárias de qualidade de água, e que, consequentemente, reflete na qualidade da água de seu principal curso d'água, o Rio Iguaçu (Figura 1).

O monitoramento quali-quantitativo foi realizado em 6 estações ao longo do rio Iguaçu (Figura 1) durante os anos de 2012 e 2013, englobando áreas ainda com pouca atividade antrópica (área a montante do ponto IG01), áreas de intenso desenvolvimento urbano (sites IG02, IG03 e IG04), e áreas a jusante do trecho mais impactado (sites IG05 e IG06).

Da série de parâmetros monitorados, foram utilizadas no presente artigo as concentrações de $\mathrm{DBO}_{5}$ (APHA, 1998: método 5210B e 4500C), OD (sonda Hach/ sensor de luminescência) e as frações de TOC, POC e DOC. A filtragem das amostras para a determinação do DOC foi realizada com membrana de acetato de celulose de $0,45 \mu \mathrm{m}$ pré-lavada com água ultrapura, com preservação ácida de $0,5 \%$ de $\mathrm{H}_{2} \mathrm{SO}_{2}$ P.A. e armazenamento a $4{ }^{\circ} \mathrm{C}$ (KNAPIK, 2014), seguido do método de combustão catalítica NDIR a $680^{\circ} \mathrm{C}\left(\mathrm{TOC}-\mathrm{V}_{\mathrm{CPH}}\right.$, Shimadzu). A vazão nas estações de controle foi determinada através da leitura de nível no momento da coleta.

\section{Proposição do modelo matemático: ROCS-Model}

O modelo proposto, denominado ROCS-Model (River Organic Carbon Simulation Model), tem como variável central de simulação a concentração de carbono orgânico para a representação da matéria orgânica na coluna d'água. O modelo foi estruturado com uma interface de entrada de dados em planilhas Excel e programação em VBA para as rotinas de geração de cargas, transporte, processos químicos e calibração, de forma a integrar os módulos de dados de monitoramento, de simulação e calibração.

Uma vez que o modelo foi estruturado também com o objetivo de aplicação em gestão de recursos hídricos, há diferentes módulos para inclusão de dados, alteração de coeficientes de

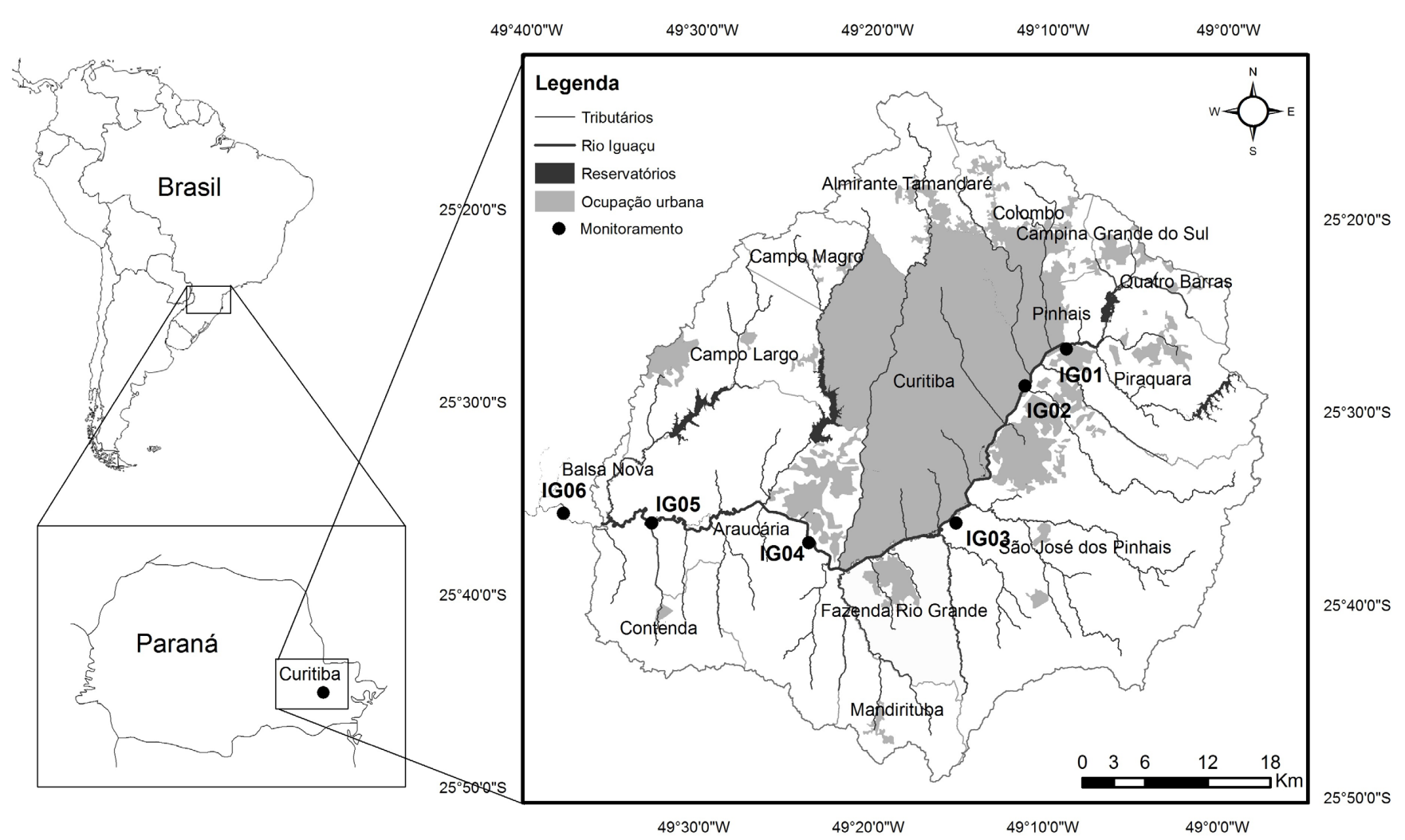

Figura 1. Mapa da Bacia do Alto Iguaçu, com a localização dos seis pontos de monitoramento (IG01 cabeceira, IG06 exutória). 
simulação, bem como de geração das cargas. Os principais módulos são: (i) geração de carga difusa com base na área e tipo de ocupação; (ii) geração de cargas pontuais de origem doméstica, industrial e de tributários; (iii) parâmetros hidráulicos; (iv) módulo de calibração; (v) módulo estatístico; entre outros.

O modelo considera a simulação unidimensional do transporte no sentido longitudinal do escoamento do rio, sendo composto por uma série de reatores bem misturados de igual comprimento (volumes de controle interligados). Para a verificação das hipóteses adotadas, foram realizadas simulações também no modelo Qual2E (BROWN; BARNWELL JUNIOR, 1987), uma vez que a representação física do modelo proposto baseia-se na mesma representação considerada pelo modelo Qual2E. A estruturação do modelo em planilhas do Excel foi baseada no modelo Qual2K (CHAPRA; PELLETIER, 2003), e permite considerar múltiplas entradas e retiradas pontuais e contribuição via fonte difusa em cada linha da planilha. A escolha dessa plataforma foi no intuito de integrar as matrizes de fontes de poluição da bacia em estudo (PORTO et al., 2007), em uma interface de fácil acesso e utilização. Assim, cada trecho do rio é representado por um número de linhas

- Carbono orgânico particulado - fração lábil (LPOC): na planilha que, de acordo com a formulação de cada módulo do programa, são geradas as respectivas cargas difusas (áreas incrementais) e cargas pontuais (grandes usuários, tributários, etc).

Com relação às variáveis de qualidade de água, o modelo simula as frações de carbono orgânico particulado lábil e refratário (LPOC e RPOC), carbono orgânico dissolvido lábil e refratário (LDOC e RDOC), conforme equações detalhadas na sequência, além de parâmetros como a DBO, o OD, série de nitrogênio e fósforo (decaimento de primeira ordem). A entrada de dados referente à relação entre os parâmetros monitorados em campo (DBO, TOC, POC e DOC) e as frações lábil e refratária (LPOC, RPOC, LDOC e RDCO), necessárias para a simulação no modelo, são apresentadas na sequência (Dados de entrada para as simulações).

Estão inclusos no modelo os processos de sedimentação e ressuspensão das frações particuladas, mineralização para formas inorgânicas, dissolução das frações particuladas para frações dissolvidas e decaimento entre as frações lábil e refratária. Um esquema dos processos considerados no balanço das frações de carbono orgânico é apresentado na Figura 2.

$$
\begin{aligned}
& \frac{d L P O C}{d t}=-\underbrace{k_{L 1} L P O C}_{\text {Sedimentação }}-\underbrace{k_{L 3} L P O C}_{\text {Mineralização }}-\underbrace{k_{L 4} L P O C}_{\text {Dissolução }}-\underbrace{k_{L 5} L P O C}_{\text {Dissolução }}-\underbrace{k_{L 6} L P O C}_{\text {Dissolução }}+\underbrace{\frac{k_{L 2}}{H}}_{\text {Ressuspensão }} \\
& L P O C=L P O C_{0} e^{-K_{L P O C} \cdot t}+\frac{k_{L 2}}{K_{L P O C} \cdot H}\left(1-e^{-K_{L P O C} \cdot t}\right)
\end{aligned}
$$

- Carbono orgânico particulado - fração refratária (RPOC):

$$
\begin{aligned}
& \frac{d R P O C}{d t}=+\underbrace{k_{L 4} \cdot L P O C}_{\text {Mineralização }}-\underbrace{k_{R l} \cdot R P O C}_{\text {Sedimentação }}-\underbrace{k_{R 3} \cdot R P O C}_{\text {Mineralização }}-\underbrace{k_{R 4} \cdot R P O C}_{\text {Decaimento }}+\underbrace{\frac{k_{R 2}}{H}}_{\text {Ressuspensão }} \\
& R P O C=R P O C_{0} e^{-K_{R P O C} \cdot t}+\frac{k_{L 4}}{\left(K_{R P O C}-K_{L P O C}\right)} L P O C_{0}\left(e^{-K_{L P O C} \cdot t}-e^{-K_{R P O C} \cdot t}\right)- \\
& \frac{k_{L 4} \cdot k_{L 2}}{\left(K_{R P O C}-K_{L P O C}\right)} L_{P O C}\left(e^{-K_{L P O C} \cdot t}-e^{-K_{R P O C} \cdot t}\right)+\frac{k_{L 4} \cdot k_{L 2}}{K_{L P O C} \cdot H}\left(1-e^{-K_{R P O C} \cdot t}\right)+\frac{k_{R 2}}{H}\left(1-e^{-K_{R P O C} \cdot t}\right)
\end{aligned}
$$

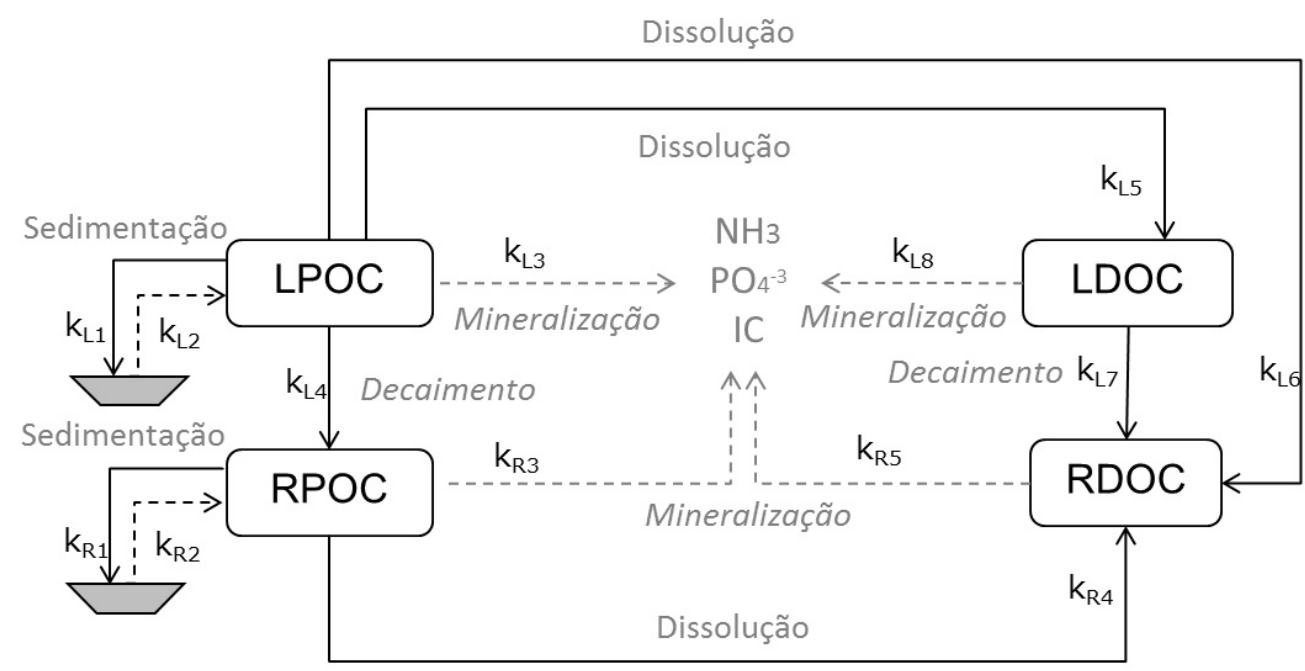

Figura 2. Representação esquemática dos processos considerados na simulação das frações de carbono orgânico. 
- Carbono orgânico dissolvido - fração lábil (LDOC):

$$
\begin{aligned}
& \frac{d L D O C}{d t}=\underbrace{k_{L 5} \cdot L P O C}_{\text {Dissolução }}-\underbrace{k_{L 7} \cdot L D O C}_{\text {Decaimento }}-\underbrace{k_{L 8} \cdot L D O C}_{\text {Mineralização }} \\
& L D O C=L D O C_{0} e^{-K_{L D O C} \cdot t}+\frac{k_{L 5}}{\left(K_{L D O C}-K_{L P O C}\right)} L P O C_{o}\left(e^{-K_{L P O C} \cdot t}-e^{-K_{L D O C} \cdot t}\right)- \\
& \frac{k_{L 5} \cdot k_{L 2}}{K_{L P O C} \cdot H \cdot\left(K_{L D O C}-K_{L P O C}\right)} L{ }^{2} C_{o}\left(e^{-K_{L P O C} \cdot t}-e^{-K_{L D O C} \cdot t}\right)
\end{aligned}
$$$$
\text { - Carbono orgânico dissolvido - fração refratária (RDOC): }
$$

$$
\begin{aligned}
& \frac{d R D O C}{d t}=\underbrace{k_{L 6} \cdot L P O C}_{\text {Dissolução }}+\underbrace{k_{L 7} \cdot L D O C}_{\text {Dissolução }}+\underbrace{k_{R 4} \cdot R P O C}_{\text {Dissolução }}-\underbrace{k_{R 5} \cdot R D O C}_{\text {Mineralização }} \\
& R D O C=R D O C_{0} e^{-k_{R 5} \cdot t}+\frac{k_{L 6}}{\left(k_{R 5}-K_{L P O C}\right)} L P O C_{o}\left(e^{-K_{L P O C} \cdot t}-e^{-k_{R 5} \cdot t}\right) \\
& +k_{L 7}\left\{\begin{array}{l}
\frac{L D O C_{O}}{\left(k_{R 5}-K_{L D O C}\right)}\left(e^{-K_{L D O C} \cdot t}-e^{-k_{R 5} \cdot t}\right) \\
+\frac{k_{L 5} \cdot L P O C_{O}}{\left(K_{L D O C}-K_{L P O C}\right) \cdot\left(k_{R 5}-K_{L P O C}\right)}\left(e^{-K_{L P O C} \cdot t}-e^{-k_{R 5} \cdot t}\right) \\
-\frac{k_{L 5} \cdot L P O C_{O}}{\left(K_{L D O C}-K_{L P O C}\right) \cdot\left(k_{R 5}-K_{L D O C}\right)}\left(e^{-K_{L D O C} \cdot t}-e^{-k_{R 5} \cdot t}\right)
\end{array}\right\} \\
& +k_{R 4}\left\{\begin{array}{l}
\frac{R P O C_{O}}{\left(k_{R 5}-K_{L P O C}\right)}\left(e^{-K_{L P O C} \cdot t}-e^{-k_{R 5} \cdot t}\right)+\frac{k_{L 4} \cdot L P O C_{o}}{\left(K_{R P O C}-K_{L P O C}\right) \cdot\left(k_{R 5}-K_{L P O C}\right)}\left(e^{-K_{L P O C} \cdot t}-e^{-k_{R 5} \cdot t}\right) \\
-\frac{k_{L 4} \cdot L P O C_{O}}{\left(K_{R D O C}-K_{L P O C}\right) \cdot\left(k_{R 5}-K_{R P O C}\right)}\left(e^{-K_{R P O C} \cdot t}-e^{-k_{R 5} \cdot t}\right) \\
-\frac{k_{L 4} \cdot k_{L 2}}{\left(K_{R P O C}-K_{L P O C}\right) \cdot K_{L P O C} \cdot H \cdot\left(k_{R 5}-K_{L P O C}\right)}\left(e^{-K_{L P O C} \cdot t}-e^{-k_{R 5} \cdot t}\right)
\end{array}\right\} \\
& +k_{R 4} \cdot\left(\frac{k_{L 4} \cdot k_{L 2}+k_{R 2} \cdot K_{L P O C}}{K_{L P O C} \cdot H}\right) \cdot\left[\frac{\left(1-e^{-k_{R 5} \cdot t}\right)}{k_{R 5}}-\frac{\left(e^{-K_{R P O C} \cdot t}-e^{-k_{R S} \cdot t}\right)}{\left(k_{R 5}-K_{R P O C}\right)}\right]
\end{aligned}
$$

em que: LPOC e RPOC são, respectivamente, as concentrações de carbono orgânico particulado lábil e refratário $(\mathrm{mg} / \mathrm{L})$; LDOC e RDOC são, respectivamente, as concentrações de carbono orgânico dissolvido lábil e refratário $(\mathrm{mg} / \mathrm{L}), \mathrm{H}$ é a profundidade $(\mathrm{m}), k_{L 1}:$ sedimentação de $\operatorname{LPOC}\left(\mathrm{d}^{-1}\right) ; k_{L 2}:$ resuspensão de $\operatorname{LPOC}\left(\mathrm{g} / \mathrm{m}^{2} \mathrm{~d}\right) ; k_{L 3}:$ mineralização de $\operatorname{LPOC}\left(\mathrm{d}^{-1}\right) ; k_{L 4}:$ decaimento de LPOC para RPOC $\left(\mathrm{d}^{-1}\right) ; k_{L 5}:$ decaimento de LPOC para LDOC $\left(\mathrm{d}^{-1}\right) ; k_{L G}$ : decaimento de LPOC para RDOC $\left(\mathrm{d}^{-1}\right)$; $k_{\mathrm{R} 1}$ : sedimentação de RPOC $\left(\mathrm{d}^{-1}\right) ; k_{\mathrm{R} 3}$ : mineralização de $\operatorname{RPOC}\left(\mathrm{d}^{-1}\right)$; $k_{\mathrm{R} 4}$ : decaimento do RPOC para RDOC $\left(\mathrm{d}^{-1}\right) ; k_{\mathrm{R} 2}$ : resuspensão do RPOC $\left(\mathrm{d}^{-1}\right) ; k_{R 5}$ : mineralização do RDOC $\left(\mathrm{d}^{-1}\right) ; k_{L 7}$ : decaimento de LDOC para RDOC $\left(\mathrm{d}^{-1}\right) ; k_{L 8}$ : mineralização do $\operatorname{LDOC}\left(\mathrm{d}^{-1}\right)$; $K_{L P O C}$ é um coeficiente auxiliar na implementação do modelo para a simulação de $\operatorname{LPOC}\left(\mathrm{K}_{\mathrm{LPOC}}=\mathrm{K}_{\mathrm{L} 1}+\mathrm{K}_{\mathrm{L} 3}+\mathrm{K}_{\mathrm{L} 4}+\mathrm{K}_{\mathrm{L} 5}+\mathrm{K}_{\mathrm{L} 0}\right)$; $K_{R P O C}$ é um coeficiente auxiliar na implementação do modelo para a simulação de RPOC $\left(\mathrm{K}_{\mathrm{RPOC}}=\mathrm{K}_{\mathrm{R} 1}+\mathrm{K}_{\mathrm{R} 3}+\mathrm{K}_{\mathrm{R} 4}\right) ; K_{L D O C}$ é um coeficiente auxiliar na implementação do modelo para a simulação de $\operatorname{LDOC}\left(\mathrm{K}_{\mathrm{LDOC}}=\mathrm{K}_{\mathrm{L} 7}+\mathrm{K}_{\mathrm{L} 8}\right)$.

\section{Algoritmo de calibração automática - PSO}

Para a calibração do modelo, foi adaptada uma rotina de otimização baseada no algoritmo de PSO - Otimização por Colônias de Partículas (KENNEDY; EBERHART, 1995; POLI;
KENNEDY; BLACKWELL, 2007; GARCIA; SCHARDONG; PORTO, 2009). Nesse processo, a calibração consiste na busca de um conjunto de coeficientes ótimos que ajustam os dados simulados à mediana dos dados observados de vazão e de concentração monitorados ao longo do Rio Iguaçu. No presente estudo foram utilizados 6 pontos de controle para a calibração do modelo (pontos de monitoramento - Figura 1). Na Equação 9 é apresentada a função objetivo utilizada na etapa da calibração do balanço de carbono orgânico.

$$
f_{O C}=\min \sum_{i=1}^{N}\left[w_{P O C}\left(P O C_{i}^{o b s}-P O C_{i}^{s i m}\right)^{2}+w_{D O C}\left(D O C_{i}^{o b s}-D O C_{i}^{s i m}\right)^{2}\right]
$$

em que: $f_{O C}$ é a função objetivo para o carbono orgânico; $P O C_{i}^{o b s}$ e $P O C_{i}^{\text {sim }}$ são, respectivamente, os valores observados e simulados de POC (mg/L); DOC ${ }_{i}^{o b s}$ e $D O C_{i}^{s i m}$ são os valores observados e simulados de DOC $(\mathrm{mg} / \mathrm{L})$, respectivamente; $w_{P O C}$ e $w_{D O C}$ são os pesos para as variáveis POC e DOC $\left(w_{P O C}=1-w_{D O C}\right)$, respectivamente; e $N$ é o número de pontos de controle (equivalente ao número de estações de monitoramento).

No caso da simulação da DBO, os limites inferior e superior dos coeficientes foi considerado de acordo com Porto et al. (2007) e Knapik (2014), enquanto os valores limites para os coeficientes do balanço de massa de carbono orgânico foram definidos pelos dados determinados experimentalmente por Knapik et al. (2015). 


\section{Dados de entrada para as simulações}

Para a estimativa das cargas de material orgânico, no caso da simulação da DBO, foi considerada como taxa de contribuição per capita o valor de DBO de $54 \mathrm{~g} /$ hab.d (WHO, 1982). Já para a simulação de carbono orgânico, e de acordo com Servais et al. (1999), para esgoto bruto, a taxa específica de contribuição per capita em termos de TOC pode variar entre 26,4 e 28,3 gC/ hab.d, sendo constituído em sua maior parte pela fração particulada, entre 19,0 e 20,7 gC/hab.d (70-76\%), e DOC de 6,5 a 8,5 gC/hab.d, com aproximadamente 60 a $75 \%$ dessa fração constituída por matéria orgânica biodegradável. Dependendo do tipo de tratamento de esgoto, a taxa específica de TOC está entre 3 e 10,8 gC/hab.d, de 2,1 a 4,9 gC/hab.d para DOC, e de 0,9 a 2,5 gC/hab.d para POC (SERVAIS et al., 1999).

Na simulação das frações de carbono orgânico, foi considerado que a fração lábil (LPOC e LDOC) é proveniente de esgoto não tratado (valores adotados de 5 e $4 \mathrm{gC} /$ hab.d, respectivamente), e a fração refratária (RPOC e RDOC) é proveniente de esgoto lançado in natura no corpo aquático (valores adotados de 20 e $8 \mathrm{gC} /$ hab.d, respectivamente). As cargas foram estimadas no módulo específico do modelo para cargas pontuais, considerando as contribuições domésticas, industriais e de tributários.

A carga doméstica foi calculada em função da distribuição da população com e sem coleta e/ou tratamento de esgoto para cada área de drenagem dos afluentes e do rio Iguaçu. Foi considerada uma vazão de produção de esgoto de 200 L/hab.dia, com 80\% de coeficiente de retorno. As respectivas eficiências de remoção das estações de tratamento de efluentes em operação, e os lançamentos de cargas industriais foram considerados de acordo com a matriz de fontes de poluição da Bacia do Alto Iguaçu (PORTO et al., 2007).

As cargas difusas foram estimadas em função das respectivas áreas de drenagem, tipo de uso e taxa de ocupação de cada tributário e do rio Iguaçu (KNAPIK, 2014). Foram considerados três tipos de uso e ocupação: urbano, agricultura e floresta. Para a DBO, foram considerados os valores de $15 \mathrm{mg} / \mathrm{L}, 5 \mathrm{mg} / \mathrm{L} \mathrm{e}$ $7 \mathrm{mg} / \mathrm{L}$ para cada tipo de uso, respectivamente, de acordo com metodologia proposta por Porto et al., 2007. Para a simulação de carbono orgânico, foi considerado que apenas a fração alóctone pedogênica contribui para a carga difusa, ou seja, apenas para as frações refratárias (RPOC e RDOC). Os valores considerados neste caso foram de $500 \mathrm{~kg} / \mathrm{km}^{2}$. ano para uso urbano, e de $1000 \mathrm{~kg} / \mathrm{km}^{2}$.ano para agricultura e floresta (MATTSSON et al., 2009).

\section{RESULTADOS E DISCUSSÃO}

De acordo com o desenvolvimento do trabalho, os resultados do presente artigo serão apresentados considerando: (i) a verificação da representação hidráulica em função da simulação analítica do modelo proposto (ROCS- Model) e a simulação numérica do modelo Qual2E (BROWN; BARNWELL JUNIOR, 1987); (ii) a calibração automática dos coeficientes do modelo proposto (rotina de otimização); e (iii) comparação entre as simulações das concentrações de carbono orgânico e da DBO para a representação da matéria orgânica no trecho do rio simulado. (i) Verificação da representação hidráulica

Conforme apresentado anteriormente, o modelo proposto foi implementado em planilhas Excel, com solução analítica para o equacionamento do modelo de transporte e balanço de massa. Múltiplas entradas e retiradas pontuais e contribuição via fonte difusa podem ser consideradas na simulação. Desta forma, a vazão total é calculada em função de contribuições difusas (vazão incremental de acordo com a área de drenagem e vazão específica, distribuída linearmente no trecho considerado) e contribuições pontuais (tributários, efluentes domésticos e industriais). Para o cálculo da vazão no trecho de rio simulado, o modelo utiliza dados de entrada tais como a área de drenagem, vazão específica, vazões nos tributários, declividades, taxas de ocupações, localização de indústrias, entre outros.

Desta forma, nas Figuras 3, 4, 5 e 6 são apresentados os resultados para as condições hidráulicas em um trecho de rio (vazão, tempo de viagem, velocidade e profundidade), comparando as simulações obtidas com o uso do modelo Qual2E (Q2E) e do modelo proposto (ROCS-Model). Conforme pode ser observado, não há diferenças entre os resultados, indicando que a discretização para a simulação da vazão e as condições hidráulicas de escoamento estão de acordo em ambos os modelos (ROCS-Model e Qual2E).

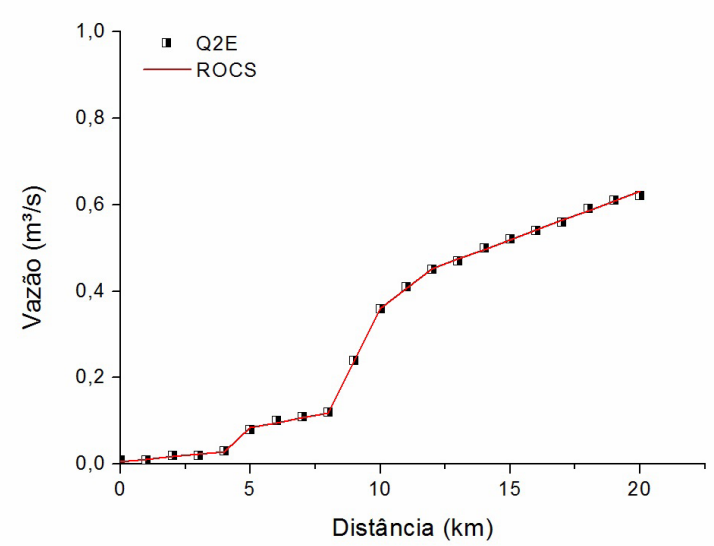

Figura 3. Comparação entre a solução numérica $(\mathrm{Q} 2 \mathrm{E})$ e do modelo proposto (ROCS) para a vazão em um trecho do rio.

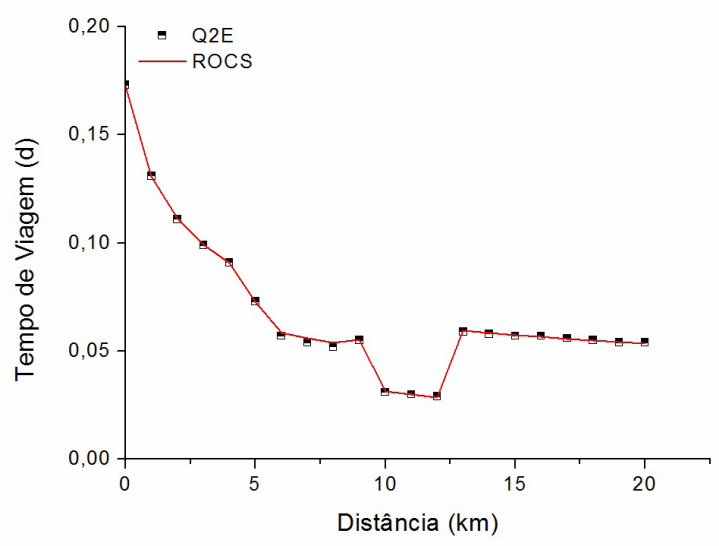

Figura 4. Comparação entre a solução numérica (Q2E) e do modelo proposto (ROCS) para o tempo de viagem em um trecho do rio. 
(ii) Calibração automática

O valor final dos coeficientes, o perfil de decaimento dos valores da função objetivo e o tempo de simulação apresentam variação em função do número de partículas do método de otimização considerado e do número total de interações aplicados na calibração com o algoritmo do PSO (KENNEDY; EBERHART, 1995; POLI; KENNEDY; BLACKWELL, 2007). Assim, considerando a minimização da função objetivo nos seis pontos de controle no rio Iguaçu, foi realizado um teste para avaliar o número de partículas e o total de interações necessários (dimensão igual a 18). As simulações foram realizadas considerando diferentes números de partículas (100, 200, 300 e 600), com variações de 10 a 50 interações. Assim como Garcia, Schardong e Porto (2009), que aplicou o algoritmo de PSO para calibração de modelos de qualidade de água, também foram adotados os valores para os coeficientes de inércia e de aceleração, respectivamente, iguais a 0,4 e 2 . A minimização da função objetivo para cada caso baseia-se na diferença entre os dados simulados e os dados observados em campo (mediana dos dados monitorados em 6 estações de controle ao longo do Rio Iguaçu), conforme exemplo apresentado na Equação 5. Os resultados são apresentados na Figura 7.

De acordo com a Figura 7, é possível observar que Quando o número de partículas é elevado, o valor final da função objetivo é alcançado em um número reduzido de interações. Enquanto a condição considerando 600 partículas pode ser realizada com menos interações, para 300 partículas, o mesmo nível do cálculo da função objetivo é alcançado com um número intermediário de interações. Assim, dependendo do tempo de processamento, é possível adotar um número intermediário de partículas e de interações.

Desta maneira, para a calibração da vazão foi padronizado um valor de 100 partículas e um número total de 50 interações (dimensão igual a 6). Para a calibração dos coeficientes referentes à simulação do balanço de massa para o carbono orgânico lábil e refratário (ROCS-Model), foi adotado um número de 250 partículas e 50 interações (dimensão igual a 78) E, para a simulação da DBO, considerada junto com a simulação de nitrogênio, fósforo e oxigênio dissolvido, foi considerado um número maior de partículas, 600, para um total de 100 interações (dimensão igual a 48) (Knapik, 2014).

Inicialmente, foi realizada a calibração da vazão simulada com os dados observados da vazão. Esta calibração se faz necessária para garantir a correspondência entre as vazões observadas em campo e as respectivas concentrações monitoradas no trecho considerado do rio Iguaçu. Conforme pode ser observado na Figura 8, com as condições adotadas, foram obtidos bons resultados no ajuste da vazão.

O mecanismo de busca do algoritmo do PSO, assim como outros algoritmos utilizados para calibração automática de modelos de qualidade de água (KONDAGESKI; FERNANDES, 2009), resulta em diferentes combinações de valores dos coeficientes considerados no balanço de massa. Desta forma, é necessário restringir de forma adequada os limites inferior e superior no algoritmo com o objetivo de obter valores que representem uma condição física, química ou biológica. Valores indicativos podem ser encontrados em literatura para determinados parâmetros ou determinados experimentalmente. Knapik (2014), por exemplo,

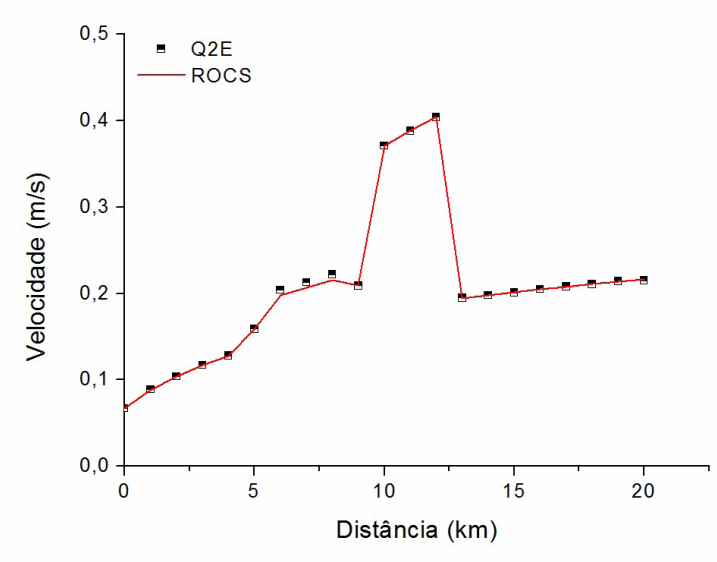

Figura 5. Comparação entre a solução numérica (Q2E) e do modelo proposto (ROCS) para a velocidade de escoamento em um trecho do rio.

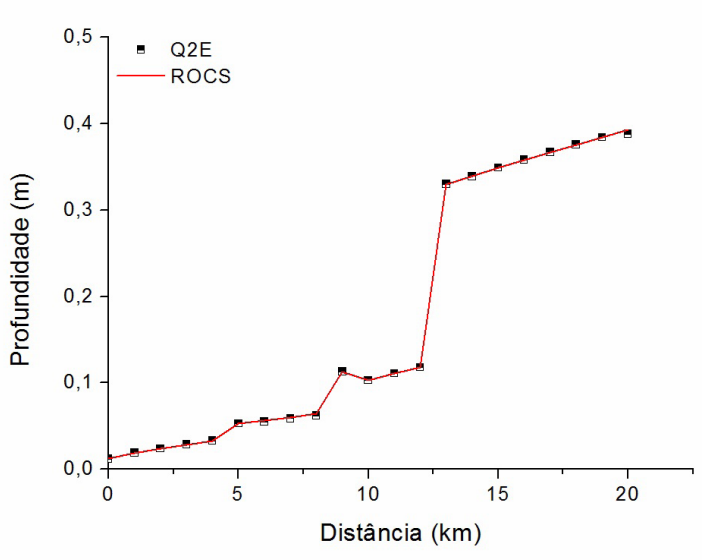

Figura 6. Comparação entre a solução numérica (Q2E) e do modelo proposto (ROCS) para a profundidade do canal em um trecho do rio.

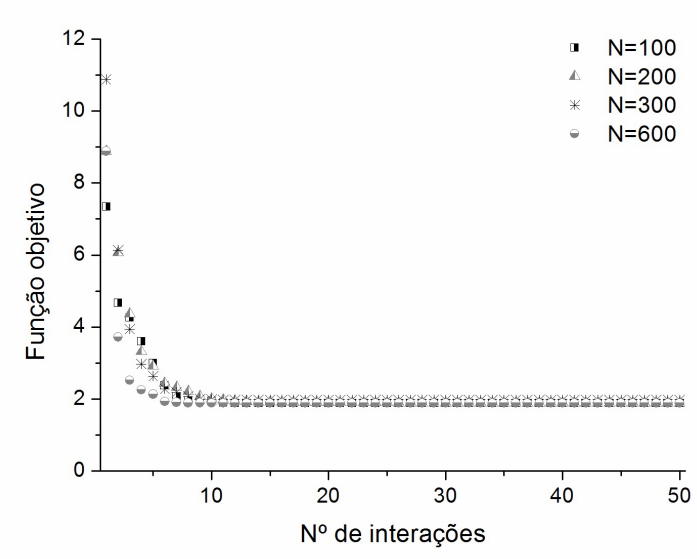

Figura 7. Variação do valor da função objetivo de acordo com o número de partículas. 
através de ensaios de biodegradação de DOC, POC e TOC no rio Iguaçu, encontrou valores para a degradação biológica na faixa de $0,07 \mathrm{~d}^{-1}$ a $0,21 \mathrm{~d}^{-1}$. Khan, Sy-Savane e Jittawattanarat (2005), obteve valores entre 0,05 e $0,08 \mathrm{~d}^{-1}$ para a decomposição biológica do DOC para águas com influência de efluentes domésticos tratados. Já Köhler et al. (2012) observou valores mais baixos para a decomposição biológica do DOC em ambientes com presença de substâncias húmicas, com valores médios de 0,0013 \pm 0,002 $\mathrm{d}^{-1}$. Conforme Knapik (2014), variações são observadas em função das características da matéria orgânica presente (maior ou menor presença de efluentes, por exemplo) e do tipo de ambiente (rio, lago, etc.). Desta forma, para este estudo de caso, considerando os coeficientes envolvidos no balanço de massa das diferentes concentrações de carbono orgânico, foram adotados como limite inferior e superior no algoritmo do PSO, respectivamente, 0,0001 e 1.
Para a calibração da simulação de carbono orgânico no modelo proposto, que considera as frações lábil e refratária do carbono orgânico dissolvido e particulado, 13 coeficientes foram calibrados com a rotina de otimização do PSO. As concentrações observadas de carbono orgânico particulado (POC, monitorado em 6 estações ao longo do rio Iguaçu), foram utilizados para avaliar a soma entre as frações lábil e refratária da matéria orgânica particulada (LPOC e RPOC), assim como as concentrações observadas de carbono orgânico dissolvido foram utilizados para a avaliar a soma entre as frações lábil e refratária da matéria orgânica dissolvida (LDOC e RDOC). A soma das simulações de POC e DOC foram calibradas com os valores observados de TOC. O resultado da calibração do TOC é apresentado na Figura 9.

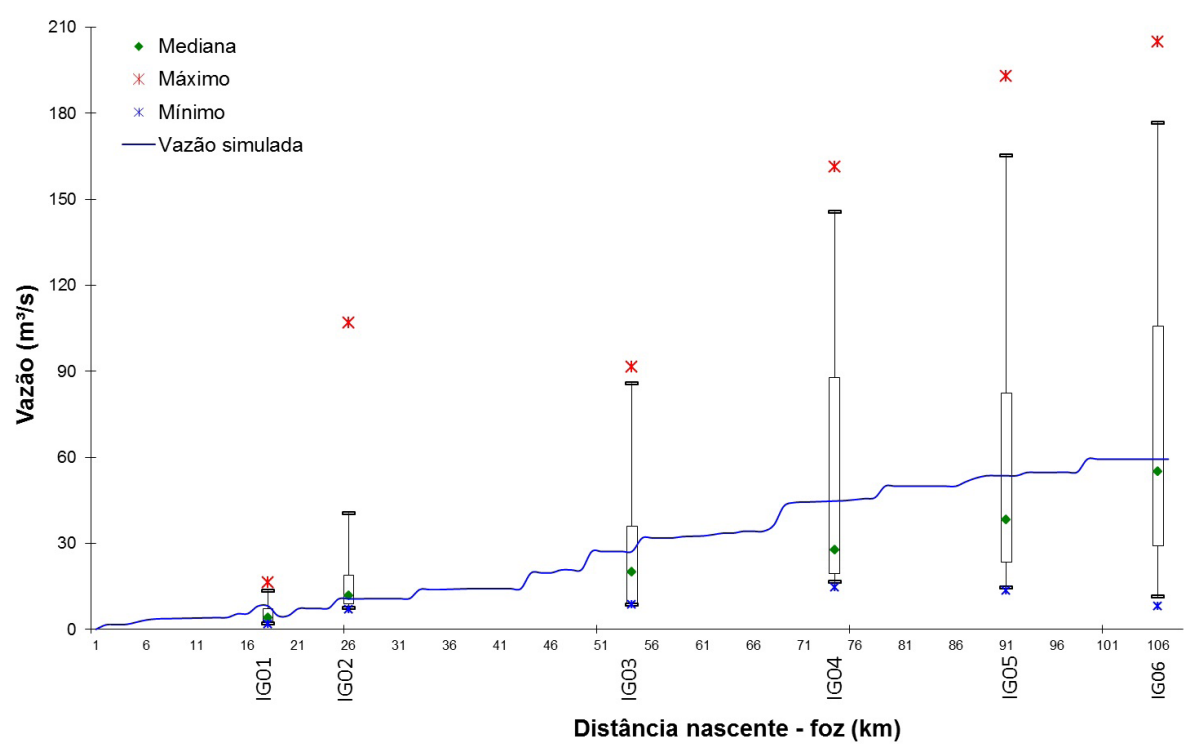

Figura 8. Resultado da calibração para a vazão, considerando os coeficientes estimados pelo algoritmo do PSO. Os valores nos box-plots representam os dados monitorados em campo (mediana, máximo e mínimo).

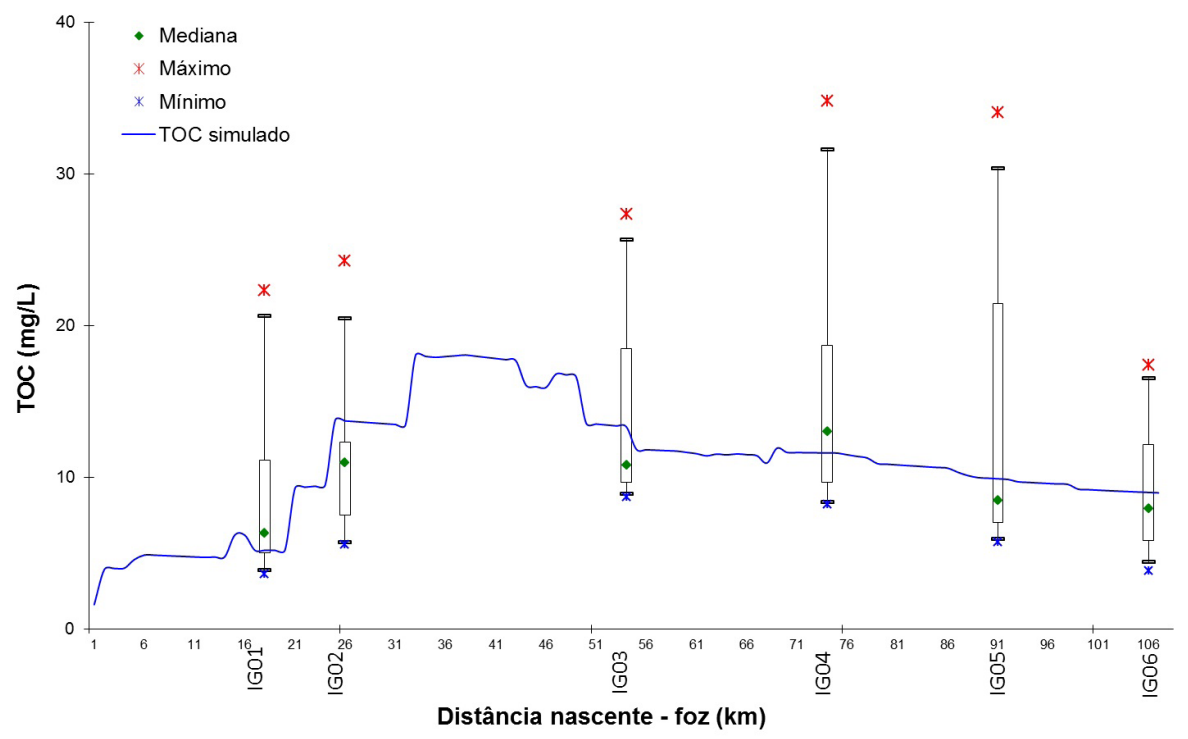

Figura 9. Resultado da calibração para a concentração de TOC, considerando os coeficientes estimados pelo algoritmo do PSO. Os valores nos box-plots representam os dados monitorados em campo (mediana, máximo e mínimo). 
Os resultados indicam (Figura 9) que a calibração dos coeficientes do balanço de massa para a concentração de TOC é adequada, atingindo-se valores simulados próximos à mediana dos dados observados durante o monitoramento de campo para a maior parte das estações de controle. Da mesma forma, a calibração automática dos coeficientes envolvidos no balanço de massa da DBO também proporcionou bons resultados, como indicado na Figura 10. Neste caso, para a calibração da simulação da DBO, foram utilizados como limites dos coeficientes no mecanismo de busca do algoritmo dados experimentais obtidos por Porto et al. (2007) e Knapik (2014), com faixa de valores para o coeficiente de desoxigenação carbonácea $\left(K_{1}\right)$ e de sedimentação $\left(K_{3}\right)$ entre 0,02 a $3,4 \mathrm{~d}^{-1}$, para as mesmas estações de controle consideradas no presente estudo. Como a simulação da DBO envolve apenas dois coeficientes (degradação biológica e sedimentação), o algoritmo se mostrou mais preciso na busca de um conjunto de valores no processo de calibração, resultando em dados simulados muito próximos à mediana dos dados observados em campo nas estações de controle. Já para a simulação do TOC, a propagação de erros nas hipóteses consideradas é maior (soma das frações determinadas em laboratório, por exemplo), o que resulta em um processo mais detalhado de busca de um conjunto de valores para os coeficientes envolvidos no balanço de massa do sistema em análise.

De acordo com as condições de simulação detalhadas anteriormente, o algoritmo do PSO convergiu para os seguintes valores dos coeficientes envolvidos no balanço de massa das frações de carbono orgânico e da DBO (Tabela 1).

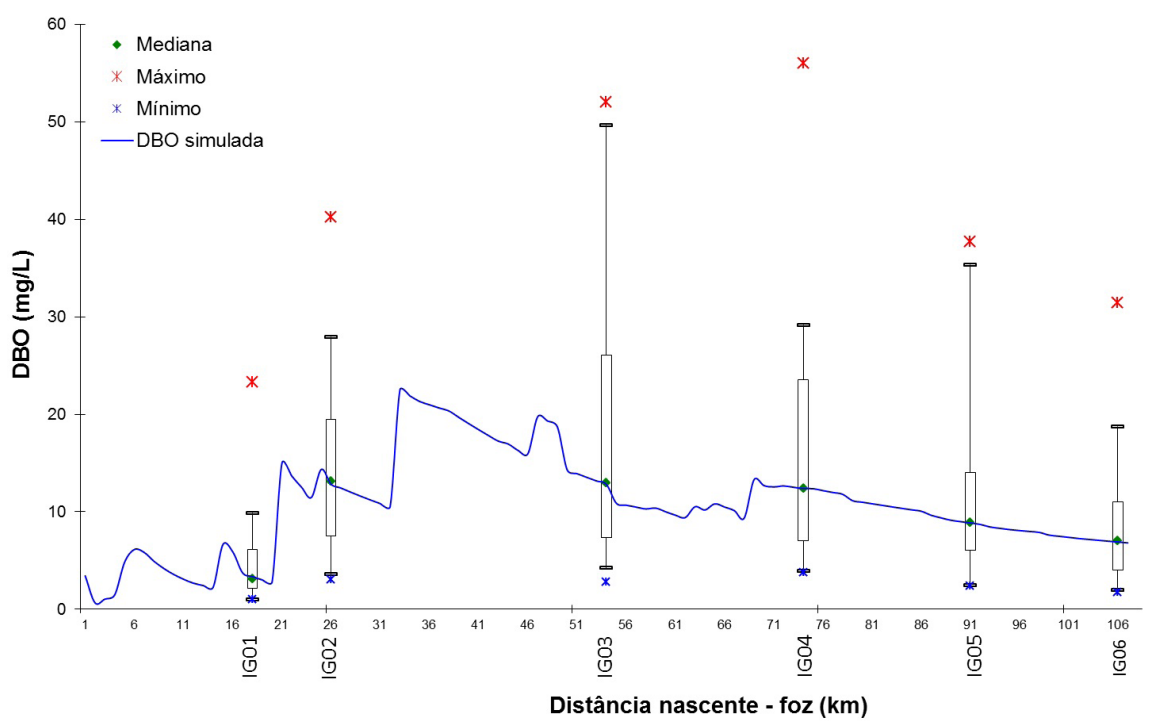

Figura 10. Resultado da calibração para a concentração de DBO, considerando os coeficientes estimados pelo algoritmo do PSO. Os valores nos box-plots representam os dados monitorados em campo (mediana, máximo e mínimo).

Tabela 1. Coeficientes obtidos pelo processo de calibração automática nos pontos de controle ao longo do trecho do rio Iguaçu.

\begin{tabular}{|c|c|c|c|c|c|c|}
\hline \multirow{2}{*}{ Coeficiente } & \multicolumn{6}{|c|}{ Pontos de controle } \\
\hline & IG01 & IG02 & IG03 & IG04 & IG05 & IG06 \\
\hline $\mathrm{K}_{\mathrm{L} 1}$ & 0,1 & 0,1 & 0,1 & 0,01 & 0,1 & 0,1 \\
\hline $\mathrm{K}_{\mathrm{L} 2}$ & 0,0873 & 0,0001 & 0,0001 & 0,0076 & 0,0001 & 0,0001 \\
\hline $\mathrm{K}_{\mathrm{L} 3}$ & 0,01 & 0,1 & 0,1 & 0,1 & 0,1 & 0,1 \\
\hline $\mathrm{K}_{\mathrm{LA}}$ & 0,1 & 0,07 & 0,1 & 0,1 & 0,1 & 0,1 \\
\hline$K_{L 5}$ & 0,1 & 0,1 & 0,1 & 0,01 & 0,1 & 0,1 \\
\hline $\mathrm{K}_{\mathrm{L} 6}$ & 0,001 & 0,1 & 0,1 & 0,079 & 0,1 & 0,1 \\
\hline $\mathrm{K}_{\mathrm{L} 7}$ & 0,1 & 0,1 & 0,0797 & 0,1 & 0,1 & 0,1 \\
\hline $\mathrm{K}_{\mathrm{L} 8}$ & 0,1 & 0,1 & 0,1 & 0,1 & 0,1 & 0,1 \\
\hline $\mathrm{K}_{\mathrm{R} 1}$ & 0,1 & 0,1 & 0,1 & 0,1 & 0,038 & 0,1 \\
\hline $\mathrm{K}_{\mathrm{R} 2}$ & 0,0880 & 0,1 & 0,1 & 0,0001 & 0,0881 & 0,0001 \\
\hline $\mathrm{K}_{\mathrm{R} 3}$ & 0,1 & 0,1 & 0,1 & 0,1 & 0,0001 & 0,1 \\
\hline $\mathrm{K}_{\mathrm{R} 4}^{\mathrm{K}}$ & 0,0001 & 0,1 & 0,1 & 0,1 & 0,1 & 0,1 \\
\hline $\mathrm{K}_{\mathrm{R} 5}^{\mathrm{K}}$ & 0,001 & 0,089 & 0,003 & 0,023 & 0,039 & 0,1 \\
\hline$K_{1}$ & 3,31 & 3,4 & 1,5 & 0,02 & 0,72 & 0,02 \\
\hline $\mathrm{K}_{3}$ & 3,4 & 2,21 & 0,02 & 1,47 & 0,04 & 0,76 \\
\hline
\end{tabular}

em que: $k_{L 1}$ : sedimentação de LPOC $\left(\mathrm{d}^{-1}\right) ; k_{I 2}$ : resuspensão de LPOC $\left(\mathrm{g} / \mathrm{m}^{2} \mathrm{~d}\right) ; k_{L 3}:$ mineralização de LPOC $\left(\mathrm{d}^{-1}\right) ; k_{L 4}:$ decaimento de LPOC para RPOC $\left(\mathrm{d}^{-1}\right)$; $k_{1}:$ decaimento de LPOC para LDOC $\left(\mathrm{d}^{-1}\right) ; k_{1}:$ decaimento de LPOC para RDOC $\left(\mathrm{d}^{-1}\right) ; k_{1}:$ decaimento de LDOC para RDOC $\left(\mathrm{d}^{-1}\right) ; k_{1}:$ mineralização do LDOC $\left(\mathrm{d}^{-1}\right)$; $k_{\mathrm{R}}$ : sedimentação de RPOC $\left(\mathrm{d}^{-1}\right) ; k_{\mathrm{R} 3}:$ mineralização de RPOC $\left(\mathrm{d}^{-1}\right) ; k_{\mathrm{R} 4}:$ decaimento do RPOC para RDOC $\left(\mathrm{d}^{-1}\right) ; k_{\mathrm{R} 2}:$ resuspensão do RPOC $\left(\mathrm{d}^{-1}\right) ; k_{\mathrm{R}}:$ mineralização do RDOC $\left(\mathrm{d}^{-1}\right) ; k_{1}$ : desoxigenação carbonácea $\left(\mathrm{d}^{-1}\right) ; k_{3}$ : sedimentação carbonácea $\left(\mathrm{d}^{-1}\right)$. 
Cabe destacar, novamente, que a calibração automática deve ser analisada com critérios, uma vez que o mecanismo de busca retorna um conjunto de valores que não necessariamente apresenta um significado físico, químico e biológico. Conforme pode ser observado nos dados apresentados na Tabela 1, o mecanismo de busca converge para o conjunto ótimo de valores dentro da faixa de valores definida no algoritmo de otimização. Outras rotinas de otimização empregadas na calibração de modelos de qualidade de água, como, por exemplo, o algoritmo genético (KONDAGESKI; FERNANDES, 2009; GARCIA; SCHARDONG; PORTO, 2009), também apresenta esta fragilidade, apesar de toda a potencialidade envolvida no processo de busca automática. São necessárias, portanto, boas estratégias de monitoramento e determinações experimentais, com abrangência espacial e temporal dos parâmetros simulados.

(iii) Simulação de carbono orgânico x DBO

Adicionalmente, foram efetuadas comparações entre a simulação da concentração das frações de carbono orgânico e da concentração de DBO com o objetivo de comparar essas duas diferentes abordagens para a representação da matéria orgânica na coluna d'água.

Os resultados da simulação analítica no trecho do Rio Iguaçu, para as diferentes frações de matéria orgânica considerada pelo modelo (dissolvida, particulada, lábil e refratária) são apresentados na Figura 11. De acordo com as hipóteses consideradas no desenvolvimento do modelo, pode-se observar a diferença de intensidade entre a fração lábil e a fração refratária, principalmente no trecho com maior atividade antrópica (após a estação IG01). A concentração de TOC permite avaliar o teor global do conteúdo orgânico na coluna d'água, enquanto as diferentes frações permitem identificar qual trecho do rio há o predomínio da fração lábil ou refratária, indicando, consequentemente, os trechos com um maior potencial de impacto na depleção da concentração de OD.
Complementarmente, os resultados da simulação das frações de carbono orgânico foram comparados com a simulação da DBO em termos de concentração molar, a fim de identificar as potencialidades e fragilidades de cada variável, bem como as áreas mais impactadas pela poluição orgânica. Para essa comparação, os resultados são apresentados na concentração molar, considerando as simulações de DBO, TOC e as respectivas concentrações lábil (LTOC) e refratária (RTOC) de carbono orgânico (Figura 12).

De uma forma holística, os ensaios de TOC, POC, DOC, DBO e DQO representem a mesma matéria orgânica na amostra analisada. Contudo, nem todos os compostos representativos são quantificados em cada um dos métodos (KNAPIK et al., 2014). Enquanto a análise de TOC representa a totalidade de carbono na amostra, sem diferenciar entre as frações lábil e refratária, o ensaio da DBO é capaz de mensurar apenas a fração biodegradável, ou seja, a parte lábil da matéria orgânica presente no ambiente analisado (THOMAS; THERAULAZ, 2007). Desta forma, uma estratégia para estimar a proporção de carbono orgânico lábil e refratário foi a de analisar a razão entre a concentração de DBO e de TOC em termos de concentração molar. A concentração molar permite comparar, na mesma unidade, a relação entre a massa de carbono assimilado e a concentração de oxigênio dissolvido consumido indiretamente na oxidação da matéria orgânica (KNAPIK et al., 2014).

Como pode ser observado na Figura 12, as curvas de DBO e LTOC apresentam um comportamento similar no trecho do rio simulado, uma vez que a DBO responde pela fração biodegradável da matéria orgânica, ou seja, a fração de fácil decomposição (lábil). Ainda, é possível identificar a relação entre as áreas mais poluídas e o trecho com maior atividade urbana (trecho intermediário da bacia entre as estações IG02 e IG04), onde estão localizados tributários

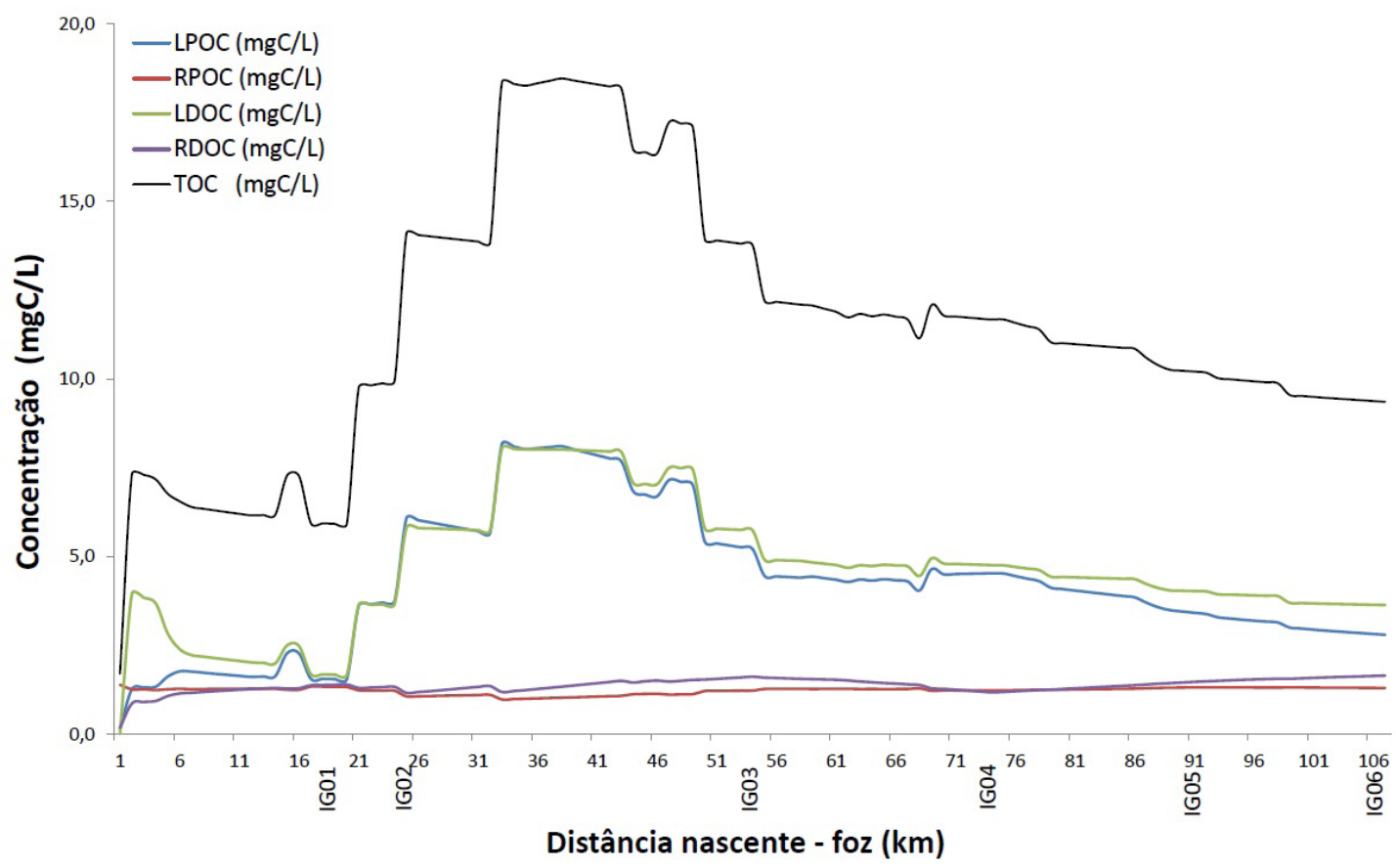

Figura 11. Comparação entre a simulação da concentração de TOC e as frações lábil particulada e dissolvida (LPOC e LDOC) e refratária (RPOC e RDOC) no rio Iguaçu. 
com elevada carga de poluição orgânica (esgotos domésticos com pouco ou nenhum tratamento).

Os resultados da simulação de carbono orgânico podem ser analisados também e função dos respectivos percentuais ao longo do trecho do rio simulado, conforme apresentado na Figura 13. De acordo com os resultados, no trecho anterior à urbanização (ponto IG01), a proporção de carbono orgânico refratário é superior, com RTOC em torno de 39\%, enquanto que a fração lábil, LTOC, permanece na faixa de $60 \%$ em média. Na parte com maior atividade antrópica, a jusante do ponto IG02, a proporção se inverte, passando a ser de $22 \%$ da fração refratária (RTOC) e de $78 \%$ para a fração lábil (LTOC). Estes resultados estão de acordo com estudos que indicam uma porcentagem entre 40 e $80 \%$ de substâncias húmicas (matéria orgânica refratária) em ambientes aquáticos não poluídos (ZUMSTEIN; BUFFLE, 1989; BENNER, 2003; FILELLA, 2009).

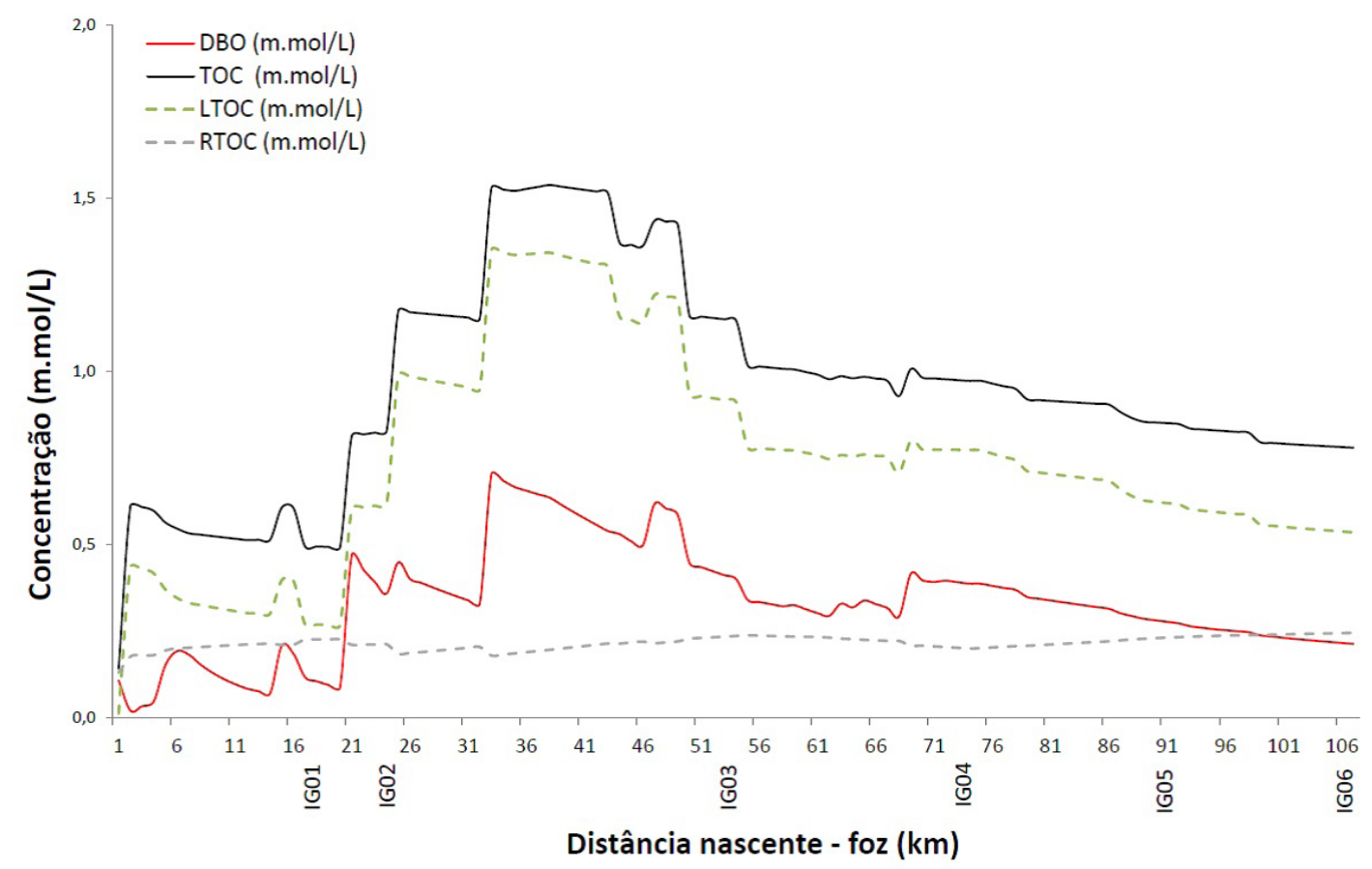

Figura 12. Resultados da simulação da concentração molar de DBO, TOC e as frações lábil (LTOC) e refratária (RTOC) no rio Iguaçu.

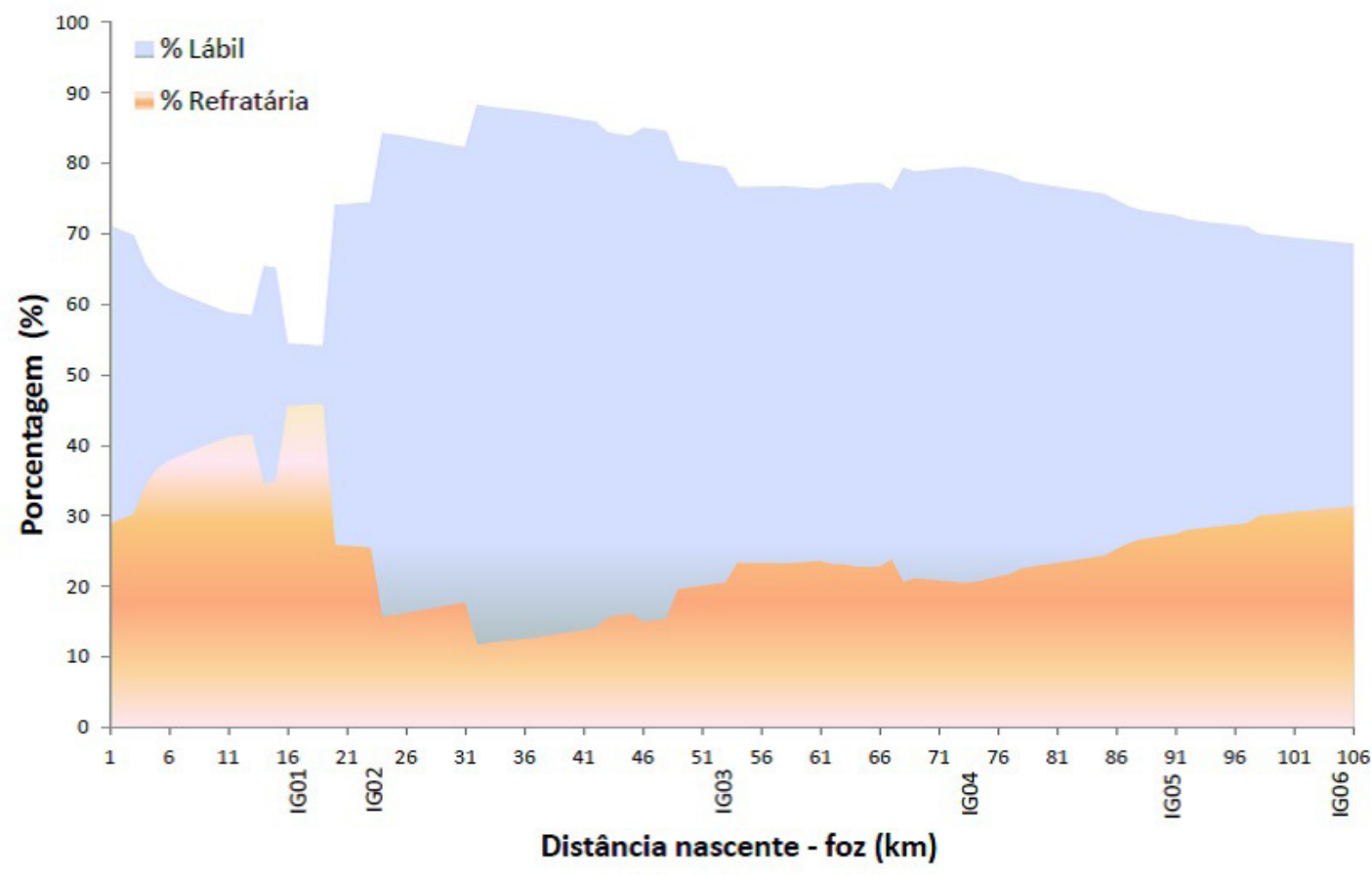

Figura 13. Comparação do percentual lábil e refratário da concentração de carbono orgânico simulada no rio Iguaçu. 
Comparando-se os resultados em termos da DBO com a concentração de TOC, pode-se observar que enquanto a DBO apresenta um rápido decaimento, o TOC resulta em um decaimento mais lento, uma vez que estão inclusos a concentração da fração refratária e os respectivos os mecanismos de transformação entre as frações de carbono orgânico. Ainda, há os processos de dissolução, no qual a fração particulada de carbono orgânico pode contribuir para a fração dissolvida. Os resultados indicam que mesmo com o predomínio da fração lábil da matéria orgânica, ainda há uma porcentagem de matéria orgânica refratária na coluna d'água.

\section{CONCLUSÃO}

De acordo com os resultados apresentados, a segmentação e modelagem das frações lábil e refratária do carbono orgânico permite uma análise mais detalhada dos processos de transformação bioquímica, bem como da identificação das áreas mais susceptíveis à deterioração da qualidade da água. No caso da bacia do Alto Iguaçu, claramente pode-se identificar essa característica após a estação IG01, onde há o predomínio de áreas com forte ocupação antrópica. De acordo com os resultados, no trecho anterior à urbanização (estação IG01) há uma maior proporção de carbono orgânico refratário (RTOC em torno de 39\%) quando comparado ao trecho com maior atividade antrópica (RTOC de $22 \%$ ), enquanto que a fração lábil ocorre o inverso (LTOC na faixa de 60\%, em média, antes do IG01 e 78\%, em média, após o IG02). Ainda, o rio apenas começa a mostrar sinais de recuperação após a estação IG05, com redução da presença de matéria orgânica lábil e de um crescente aumento do remanescente de matéria orgânica refratária.

A rotina de otimização utilizada para a calibração automática do modelo (PSO) mostrou-se bastante eficiente, mesmo para um grande número de coeficientes, reduzindo o tempo usualmente utilizado em ajustes por tentativa e erro. Uma questão importante é a definição dos limites de busca dos valores para os coeficientes envolvidos na modelagem. Assim como outros algoritmos evolutivos, o processo de otimização encontra um conjunto de valores que minimizam a função objetivo. Contudo, não necessariamente esses valores resultam com um significado físico, químico e biológico. No presente estudo, a utilização dos dados monitorados em 6 estações de controle ao longo do Rio Iguaçu, bem como de dados experimentais para referência nos limites mais adequados, foram essenciais para a boa calibração obtida.

Considerando a aplicabilidade em gestão de recursos hídricos na questão da modelagem matemática para a simulação da qualidade da água, para a utilização do carbono orgânico como variável de estado, como no caso do modelo ROCS-Model, novas estratégias que incluam o monitoramento deste parâmetro são necessárias. Não há, contudo, uma normatização referente às análises das diferentes frações, bem como dos valores de referência para fins de gestão e fiscalização, ou seja, ainda não há um consenso sobre quais níveis de concentração são relevantes e como comparar esses níveis com valores conhecidos de DBO ou DQO. Desta forma, destaca-se a potencialidade da abordagem aqui apresentada, mas, com implicações inerentes aos dados de entrada no modelo. Contudo, comparando-se com outros modelos que também consideram a matéria orgânica de forma mais completa do que somente o balanço de massa para a DBO, o modelo proposto tem vantagens em ser menos complexo da questão dos dados de entrada e número de parâmetros e coeficientes necessários na implementação desses modelos. Esta questão é fundamental para ser um modelo viável de aplicação pelos órgãos gestores de bacias, em especial na problemática envolvida em bacias urbanas.

Complementarmente, outra vantagem do modelo proposto é o seu fácil uso e acesso, uma vez que é todo implementado em planilhas Excel. Os módulos contemplados no modelo permitem ao usuário integrar a matriz de fontes de poluição diretamente como dados de entrada, facilitando a simulação para diversas bacias. O modelo em questão encontra-se disponível para uso no meio acadêmico, e está em estudo o desenvolvimento de uma segunda versão com automatização de rotinas complementares para tornar ainda mais acessível a aplicação em outras bacias, de modo a poder contribuir para um melhor entendimento da dinâmica da poluição orgânica e consolidar estratégias de planejamento e gestão da qualidade da água.

Finalmente, como vantagens da determinação das concentrações das frações de carbono orgânico (TOC, POC e DOC), podem ser listadas a menor subjetividade, maior rapidez e maior confiabilidade nas análises quando comparado aos parâmetros tradicionalmente utilizados em monitoramento e modelagem da qualidade da água, tais como a DBO. Contudo, ainda são necessários mais avanços para o monitoramento das frações lábil e refratária, e, principalmente, na análise conjunta com outras técnicas de análise como, por exemplo, a espectroscopia de fluorescência e de ultravioleta visível. Ainda, estes parâmetros não são contemplados pela legislação brasileira, fazendo com que seja necessária a correspondência com os dados monitorados e simulados de DBO para fins de fiscalização e controle.

Considerando bacias com intensa atividade urbana como a Bacia do Alto Iguaçu e os resultados aqui apresentados, pode-se afirmar que as características do modelo proposto são fundamentais para a consolidação de estratégias de monitoramento e modelagem com foco na gestão da qualidade da água.

\section{AGRADECIMENTOS}

Os autores agradecem o apoio financeiro de MCT/CNPq $\mathrm{n}^{\circ}$ 4/2010 (471456/2010-1); a bolsa de doutorado do primeiro autor (MCT/ CNPq/ CT-Hidro no 22/2009 (processo 142130/2010-9) \& Capes-Fulbright (DRI/CGCI n ${ }^{\circ}$ 040/2010)). Esta pesquisa também foi parcialmente financiada por MCT/FINEP/CT-HidroGRH 01/2004 n o 014100000 e MCT/FINEP/CT-Hidro-IGRH $01 / 2007$.

\section{REFERÊNCIAS}

APHA - AMERICAN PUBLIC HEALTH ASSOCIATION. Standard methods for the examination of water and wastewater. 20. ed. Washington, 1998.

BENNER, R. Molecular indicator of the bioavailability of dissolved organic matter. In: FINDLAY, S. E. G.; SINSABAUGH, R. L. Aquatic ecosystems: interactivity of dissolved organic matter. Nova York: Elsevier, 2003. cap. 5, p. 121- 137. 
BROWN, L. C.; BARNWELL JUNIOR, T. O. The enhanced stream water quality model QUAL2E and QUAL2EUNCAS: computer program documentation and user manual. Athens: United States Environmental Protection Agency, 1987. 189 p.

CHAPRA, S. C. Organic carbon and surface water quality modeling. Progress in Environmental Science, v. 1, n. 1, p. 49-70, 1999.

CHAPRA, S. C.; PELLETIER, G. QUAL2K: a modeling framework for simulation river and stream water quality: documentation and user's manual. Medford: Tufts University, 2003. 121 p.

CLOUGH, J. S. A. QUATOX (R.3.1 plus): modeling environmental fate and ecological effects in aquatic ecosystems: user's manual. Washington: United States Environmental Protection Agency, 2014. 109 p. (v. 1).

COLE, T. W.; WELLS, S. A. Ce-QUAL-W2: a two-dimensional laterally averaged, hydrodynamic and water quality model, version 3.6. Washington: U.S. Army Corps of Engineers, 2008. 634 p.

COMBER, S. D. W.; GARDNER, M. J.; GUNN, A. M. Measurement of absorbance and fluorescence as potential alternatives to BOD. Environmental Technology, v. 17, n. 7, p. 771-776, 1996. http://dx.doi. org/10.1080/09593331708616444.

DELTARES. D-Water Quality: versatile water quality modelling in $1 \mathrm{D}, 2 \mathrm{D}$ or $3 \mathrm{D}$ systems including physical, (bio)chemical and biological processes: user manual. Delft, 2014. 394 p.

EVANS, C. D.; MONTEITH, D. T.; COOPER, D. M. Long-term increases in surface water dissolved organic carbon: observations, possible causes and environmental impacts. Environmental Pollution, v. 137, n. 1, p. 55-71, 2005. http://dx.doi.org/10.1016/j. envpol.2004.12.031. PMid:15944040.

FILELLA, M. Freshwaters: which NOM matters? Environmental Chemistry Letters, v. 7, n. 1, p. 21-35, 2009. http://dx.doi.org/10.1007/ s10311-008-0158-x.

GARCIA, J. I. B.; SCHARDONG, A.; PORTO, R. L. Desempenho de algoritmos evolucionários na calibração de modelo de qualidade da água. In: SIMPÓSIO BRASILEIRO DE RECURSOS HÍDRICOS, 18., 2009, Campo Grande, MS. Anais... Campo Grande: Associação Brasileira de Recursos Hídricos, 2009. CD-ROM.

JOUANNEAU, S.; RECOULES, L.; DURAND, M. J.; BOUKABACHE, A.; PICOT, V.; PRIMAULT, Y.; LAKEL, A.; SENGELIN, M.; BARILLON, B.; THOUAND, G. Methods for assessing biochemical oxygen demand (BOD): a review. Water Research, v. 49, p. 62-82, 2014. http://dx.doi.org/10.1016/j. watres.2013.10.066. PMid:24316182.

KARLSSON, O. M.; RICHARDSON, J. S.; KIFFNEY, P. M. Modelling organic matter dynamics in headwater streams of south-werstern Britsh Columbia, Canada. Ecological Modelling, v. 183, n. 4, p. 463-476, 2005. http://dx.doi.org/10.1016/j. ecolmodel.2004.08.022.
KENNEDY, J.; EBERHART, R. C. Particle swarm optimization. Proceedings of IEEE International Conference on Neural Networks, v. 4, p. 1942-1948, 1995. http://dx.doi.org/10.1109/ICNN.1995.488968.

KHAN, E.; SY-SAVANE, O.; JITTAWATTANARAT, R. Application of commercial biochemical oxygen demand inocula for biodegradable dissolved organic carbon determination. Water Research, v. 39, n. 19, p. 4824-4834, 2005. http://dx.doi. org/10.1016/j.watres.2005.09.036. PMid:16278006.

KNAPIK, H. G. Organic matter characterization and modelling in polluted rivers for water quality planning and management. 2014. 318 f. Tese (Doutorado em Engenharia de Recursos Hídricos e Ambiental) - Universidade Federal do Paraná, Curitiba, 2014.

KNAPIK, H. G.; FERNANDES, C. V. S.; AZEVEDO, J. C. R.; PORTO, M. F. A. Applicability of fluorescence and absorbance spectroscopy to estimate organic pollution in rivers. Environmental Engineering Science, v. 31, n. 12, p. 653-663, 2014. http://dx.doi. org/10.1089/ees.2014.0064. PMid:25469076.

KNAPIK, H. G.; FERNANDES, C. V.; AZEVEDO, J. C.; SANTOS, M. M.; DALL'AGNOL, P.; FONTANE, D. G. Biodegradability of anthropogenic organic matter in polluted rivers using fluorescence, UV, and BDOC measurements. Environmental Monitoring and Assessment, v. 187, n. 3, p. 1-15, 2015. PMid:25666647.

KNAPIK, H.; FERNANDES, C.; PICKBRENNER, K.; PORTO, M.; BASSANESI, K. Qualidade da Água da Bacia do Rio Iguaçu: diferenças conceituais entre os modelos Qual2E e Qual2k. Revista Brasileira de Recursos Hidricos, v. 16, n. 2, p. 75-88, 2011. http:// dx.doi.org/10.21168/rbrh.v16n2.p75-88.

KÖHLER, B.; VON WACHENFELDT, E.; KOTHAWALA, D.; TRANVIK, L. Reactivity continuum of dissolved organic carbon decomposition in lake water. Journal of Geophysical Research, v. 117, n. G1, p. 1-14, 2012.

KONDAGESKI, J. H.; FERNANDES, C. V. S. Calibração de modelo matemático de qualidade da água utilizando algoritmo genético: estudo de caso do rio Palmital, PR. Revista Brasileira de Recursos Hidricos, v. 14, n. 1, p. 63-73, 2009. http://dx.doi. org/10.21168/rbrh.v14n1.p63-73.

LEENHEER, J. A.; CROUÉ, J. P. Characterizing dissolved aquatic organic matter. Environmental Science \& Technology, v. 37, n. 1, p. 1826, 2003. http://dx.doi.org/10.1021/es032333c. PMid:12542280.

MATTSSON, T.; KORTELAINEN, P.; LAUBEL, A.; EVANS, D.; PUJO-PAY, M.; RÄIKE, A.; CONAN, P. Export of dissolved organic matter in relation to land use along a European climatic gradient. The Science of the Total Environment, v. 407, n. 6, p. 19671976, 2009. http://dx.doi.org/10.1016/j.scitotenv.2008.11.014. PMid:19064278.

MOPPER, K.; ZHOU, X.; KIEBER, R. J.; KIEBER, D. J.; SIKORSKI, R. J.; JONES, R. D. Photochemical degradation of dissolved organic carbon and its impact on the oceanic carbon 
cycle. Nature, v. 353, n. 6339, p. 60-63, 1991. http://dx.doi. org/10.1038/353060a0.

MOSTOFA, K.M.G.; LIU, C.; MOTTALEB, M.A.; WAN, G.; OGAWA, H.; VIONE, D.; YOSHIOKA, T.; WU, F. Dissolved organic matter in natural waters. In: MOSTOFA, K. M. G.; YOSHIOKA, T.; MOTTALEB, M. A.; VIONE, D. Photobiogeochemistry of organic matter: principles and practices in water environments. Berlin: Environmental Science and Engineering, Springer-Verlag Berlin, 2013, cap. 1, p. 1-137.

OUYANG, Y. Simulating dyamic load of naturally occurring TOC from watershed into a river. Water Research, v. 37, n. 4, p. 823-832, 2003. http://dx.doi.org/10.1016/S0043-1354(02)003895. PMid:12531264.

POLI, R.; KENNEDY, J.; BLACKWELL, T. Particle Swarm Optimization: an overview. Swarm Intelligence, v. 1, n. 1, p. 33-57, 2007. http://dx.doi.org/10.1007/s11721-007-0002-0.

PORTO, M. F. A.; FERNANDES, C. V. S.; KNAPIK, H. G.; FRANÇA, M. S.; BRITES, A. P. Z.; MARIN, M. C. F. C.; MACHADO, F. W.; CHELLA, M. R.; SÁ, J. F.; MASINI, L. Bacias críticas: bases técnicas para a definição de metas progressivas para seu enquadramento e a integração com os demais instrumentos de gestão. Curitiba: Departamento de Hidráulica e Saneamento, UFPR, 2007. (FINEP/ CT-HIDRO). Projeto concluído.

SERVAIS, P.; GARNIER, J.; DEMARTEAU, N.; BRION, N.; BILLEN, G. Supply of organic matter and bacteria to aquatic ecosystems through waste water effluents. Water Research, v. 33, n. 16, p. 3521-3531, 1999. http://dx.doi.org/10.1016/S00431354(99)00056-1.

SHANAHAN, P.; HENZE, M.; KONCSOS, L.; RAUCH, W.; REICHERT, P.; SOMLYODY, L.; VANROLLEGHEM, P. River Water Quality Modelling II: problem of the art. Water Science and Technology, v. 38, n. 11, p. 245-252, 1998. http://dx.doi.org/10.1016/ S0273-1223(98)00661-1.

SORRIBAS, M. V.; COLLISCHONN, W.; MARQUES, D. M.; FRAGOSO JÚNIOR, J. R.; CASTRO, N.; SOUZA, R. Modelagem distribuída de carbono em bacias hidrográficas. Revista Brasileira de Recursos Hidricos, v. 17, n. 3, p. 225-240, 2012. http://dx.doi. org/10.21168/rbrh.v17n3.p225-240.

THOMAS, O.; THERAULAZ, F. Agregate organic constituints. In: THOMAS, O.; BURGESS, C. UV -visible spectrophotometry of water and wastewater. Amsterdam: Elsevier, 2007. cap. 4, p. 89-113. (Techniques and Instrumentation in Analytical Chemistry, 27).

WEISSENBERGER, S.; LUCOTTTE, M.; HOUEL, S.; SOUMIS, N.; DUCHEMIN, E.; CANUEL, R. Modeling the carbon dynamics of the La Grande Hydroelectric complex in northern Quebec. Environmental Modeling, v. 221, n. 4, p. 610-620, 2010. http://dx.doi. org/10.1016/j.ecolmodel.2009.10.037.

WESTPHAL, K. S.; CHAPRA, S. C.; SUNG, W. Modeling TOC and UV-254 absorbance for reservoir planning and operation. Journal of the American Water Resources Association, v. 40, n. 3, p. 795809, 2004. http://dx.doi.org/10.1111/j.1752-1688.2004.tb04459.x.

WHO - WORLD HEALTH ORGANIZATION. Rapid assessment of sources of air, water and landpollution. Genebra, 1982. WHO Offset Publication, n. 62.

ZUMSTEIN, J.; BUFFLE, J. Circulation of pedogenic and aquagenic organic matter in an eutrophic lake. Water Research, v. 23, n. 2, p. 229-239, 1989. http://dx.doi.org/10.1016/0043-1354(89)90047-X.

\section{Contribuição dos autores}

Heloise Garcia Knapik: Contribuiu na pesquisa bibliográfica e caracterização da área de estudo, coleta, tratamento e análise de dados, elaboração da metodologia e desenvolvimento do modelo, discussão de resultados e elaboração do texto.

Cristovão Vicente Scapulatempo Fernandes: Contribuiu na orientação e concepção do trabalho, atividades de monitoramento de campo, análise de dados, discussão de resultados e elaboração do texto.

Júlio César Rodrigues de Azevedo: Contribuiu na orientação e concepção do trabalho, atividades de monitoramento de campo, análise de dados, discussão de resultados e elaboração do texto. 